\title{
The Improved PASTd and ESPRIT in Power System Harmonic Estimation Application
}

\author{
Feng Liu, Ligong Sun* and Zhihao Cheng \\ Electrical Engineering College, Henan University of Science and Technology, Luoyang 471023, China \\ *Corresponding author
}

\begin{abstract}
The paper analyzes the current power system harmonic estimation algorithms which have a considerable number of shortages. In order to improve detection precision and reduce the computational complexity, we can combine the ESPRIT algorithm with improved PASTd algorithm, consequently, achieve the rapid estimation of harmonic frequency. Both theoretical analysis and simulation results show that the algorithm is characterized by better frequency resolution performance and anti-interference ability.
\end{abstract}

Keywords—harmonic estimation; PASTd; MDL; ESPRIT

\section{INTRODUCTION}

Nowadays, being extensively used, electric energy covers almost every aspect of our daily life. The signal that frequency should be a multiple of $50 \mathrm{~Hz}$ in the power system is defined as harmonic. For one thing, harmonic poses a great deal of potential threats to the security, stability and economic development of power system; for another, it brings about great hidden hazards to the power system. In order to bring the increasingly serious harmonic pollution under control as well as improve power quality, it is meaningful for us to extract harmonics and harmonic components from the signal. Therefore, a wide range of national and international experts concentrate efforts on the method of fast detection for harmonics which have made significant achievements, for instance, fast fourier transform (FFT) [1,2], wavelet transform (Wavelet) [3], multiple signal classification algorithm [4] and ESPRITE algorithm.

As a main tool for harmonic analysis, fast fourier trans form (FFT) is noted for simple calculation and high velocity. However, spectrum leakage may be inevitable when interharmonic exists in the signal. Although the results can be compensated by means of windowed and interpolation, requirements for the length of window functions should be taken into account. What's more, the real-time of parameter estimation tends to be inaccurate. Wavelet transform has the edge on localization, however, there have existed aliasing in frequency domain among wavelet functions that have different scales. If harmonic components that have the similar frequency are included in the signal, it is impossible to detect harmonics and interharmonics with unerring precision.

The basic principle of modern spectrum estimation is subspace decomposition, which mainly consists of MUSIC and ESPRIT algorithm. Subspace decomposition is defined as follows: firstly, in order to acquire signal subspace and noise subspace that must be characterized by orthogonal complementarity, the eigenvalue and singular value should be decomposed for autocorrelation matrix or covariance matrix of the signal. Then, exact spectrum estimation tends to be available by means of its orthogonality and rotational invariance.

There has a common feature in the preceding algorithm that is Eigenvalue Decomposition. It is well known that Eigenvalue Decomposition is noted for heavy computation, highly complex computation and difficulty in realization. Additionally, the preceding algorithm is a kind of batch processing, that is, the algorithm should be used only once after all the observational datum are available. It is obvious that such an algorithm applies to the signal that parameters or statistics vary from time to time. In order to solve this problem, some experts propose a new method that subspace iteration method takes place of feature subspace decomposing algorithm, which is typical of projection approximation subspace tracking algorithm [5].

Signal subspace matrix can be obtained by keeping the objective function to a minimum in PASTd algorithm. There is no need of ceaseless Eigenvalue Decomposition for the covariance matrix. What's more, characterized by better convergent speed and error property, PASTd algorithm can reduce the calculated amount for Eigenvalue Decomposition.

In this paper, we will combine the ESPRIT algorithm with the improved PASTd algorithm. To begin with, in order to make the PASTd algorithm convergent as soon as possible, initial value required by PASTd algorithm can be obtained by Eigenvalue Decomposition of the top several data vector during the decomposition of the subspace. Secondly, subspace should be in the Schmidt orthogonality. Then, subject to the MDL rule, the rank of subspace can be available. Meanwhile, optimal signal subspace can be obtained by determining whether the interference changes and taking measures. From the above analysis, we may arrive at the conclusion that this algorithm, which combines with the improved PASTd algorithm to solve the eigenvector from the signal subspace, can push the calculated complexity into smaller and get the harmonic frequency exactly.

All the above mentioned factors should be taken into consideration in the paper. In the first place, the basic principle for harmonic frequency estimation based on the ESPRIT algorithm will be discussed. In the next place, the paper proposes a new method that subspace iteration method takes place of feature subspace decomposing algorithm. What's more, the estimation method of signal order " $r$ " will be introduced. At 
last, harmonic estimation algorithm will be verified by means of numerical simulation.

\section{SIGNAL MODEL}

With respect to the power system of $50 \mathrm{~Hz}$ power frequency, the signal that frequency should be multiple of $50 \mathrm{~Hz}$ in the spectrum, which can be obtained by the Fourier decomposition of the voltage and current, is defined as harmonic. Hence, sampled signal that included in noise, power frequency and harmonics of the voltage and current signal in the power system can be expressed as

$$
x(n)=\sum_{i=1}^{M} A_{i} \cos \left(2 \pi f_{i} n+\phi_{i}\right)+e(n)
$$

Where $x(n)$ represents sampled signal that consists of white noise and combined sine-wave signal, $A_{1}$ is harmonic amplitude,

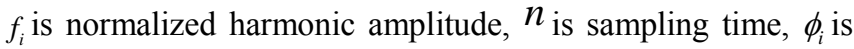
initial phase of harmonics, $e(n)$ represents white Gaussian noise of complex value that zero mean and variance equal to $\delta_{e}^{2}, M$ is number of harmonics, $\mathrm{N}$ is sampling number.

In brief, $\omega=2 \pi f$, if $x(n)$ is complex frequency signal, the equation (1) is rewritten as

$$
x(n)=\sum_{i=1}^{M} A_{i} e^{j \omega_{i} n}+e(n)
$$

Where $A_{i}$ and $\omega_{i} \in(-\pi, \pi)$ respectively represent complex amplitude and frequency of the i-th harmonic.

In brief, the new variables $\mathrm{y}(\mathrm{n})=\mathrm{x}(\mathrm{n}+1)$ and $\mathrm{m} \times 1$ may be defined as follows:

$$
X(n)=[x(n), x(n+1), \cdots, x(n+m-1)]^{T}
$$

Then, substituting the above symbols into the equation (4) yields

$$
X(n)=A S(n)+E(n)
$$

Where $A=\left[a\left(\omega_{1}\right), a\left(\omega_{2}\right), \cdots, a\left(\omega_{M}\right)\right]$,

$$
\begin{gathered}
\mathrm{a}\left(\omega_{i}\right)=\left[1, e^{j \omega_{i} T_{s}}, \cdots, e^{j(m-1) \omega_{i} T_{s}}\right]^{T} \\
S(n)=\left[A_{1} e^{j\left(n \omega_{1}\right)}, A_{2} e^{j\left(n \omega_{2}\right)}, \cdots, A_{M} e^{j\left(n \omega_{M}\right)}\right]^{T} \\
E(n)=[e(n), e(n+1), \cdots, e(n+m-1)]^{T}
\end{gathered}
$$

In brief, rotating factor matrix will be introduced as follows: $\Phi=\operatorname{diag}\left[e^{j \omega_{1}}, e^{j \omega_{2}}, \cdots, e^{j \omega_{M}}\right]$ Obviously, (1) $\Phi$ represents the unitary matrix, that is

(2) $S(n+1)=\Phi S(n)$ Hence,

$$
\Phi \Phi^{\mathbf{H}}=\Phi^{\mathbf{H}} \Phi=I
$$

$$
\begin{aligned}
& Y(n)=X(n+1)=A S(n+1)+E(n+1) \\
& =\operatorname{A\Phi S}(n)+E(n+1)
\end{aligned}
$$

\section{THE BASIC PRINCIPLE FOR HARMONIC FREQUENCY ESTIMATION BASED ON THE ESPRIT ALGORITHM}

With respect to observational vector $X(n)$, the crossvariance matrix is $R_{X X}, X(n), Y(n)$ of autocorrelation matrix can be written as $R_{X Y}$.

The eigenvalue decomposition of autocorrelation matrix and cross-variance matrix is as follows:

$$
\begin{array}{r}
R_{X X}=E\left\{X(n) X^{H}(n)\right\}=\mathrm{APA}^{\mathrm{H}}+\sigma_{e}^{2} \mathrm{I}_{M} \\
R_{X Y}=E\left\{X(n) Y^{H}(n)\right\}=\mathrm{AP} \Phi^{H} \mathrm{~A}^{H}+\sigma_{e}^{2} \mathrm{Z}
\end{array}
$$

Where $P=E\left\{S(n) S^{H}(n)\right\} \quad, \quad Z=\left[\begin{array}{cccc}0 & & & 0 \\ 1 & 0 & & \\ & \ddots & \ddots & \\ 0 & & 1 & 0\end{array}\right]$, $\sigma_{e}^{2} \mathrm{I}_{M}=E\left\{E(n) E^{H}(n)\right\}$,

$\sigma_{e}^{2} Z=E\left\{E(n) E^{H}(n+1)\right\}$, under the leading diagonal, all the components on the diagonal are 1 , the others equal to 0 .

The eigenvalue decomposition of $R_{X X}$ is directly written as:

$$
\begin{aligned}
& R_{X X}=\sum_{i=1}^{M} \lambda_{i} e_{i} e_{i}^{H} \\
& =\sum_{i=1}^{D} \lambda_{i} e_{i} e_{i}^{H}+\delta_{e}^{2} \sum_{i=D+1}^{M} \lambda_{i} e_{i} e_{i}^{H} \\
& =U \Lambda U^{T}=U_{s} \Lambda_{s} U_{s}{ }^{T}+U_{n} \Lambda_{n} U_{n}{ }^{T} \\
& \text { From } R_{X X}=U \Lambda U^{T}=\mathrm{APA}^{\mathrm{H}}+\sigma_{e}^{2} \mathrm{I}_{M},
\end{aligned}
$$

Then, $\mathrm{APA}^{\mathrm{H}}=U \Lambda U^{T}-\sigma_{e}^{2} \mathrm{I}_{M}=U\left(\Lambda-\sigma_{e}^{2} \mathrm{I}_{M}\right) U^{T}$

Based on the above, the harmonic frequency estimation can be obtained by getting the eigenvalue of pencil of matrix $\left(C_{X X}, C_{X Y}\right)$, yielding $f_{i}=\frac{\omega_{i}}{2 \pi}=\frac{1}{2 \pi} \arctan \left(\frac{\operatorname{Im}\left(\gamma_{i}\right)}{\operatorname{Re}\left(\gamma_{i}\right)}\right)$.

\section{THE ALGORITHM BASED ON THE COMBINATION OF THE IMPROVED PASTD AND ESPRIT ALGORITHM}

The fundamental view of PASTd algorithm is that converts the issue of eigenvector estimation into the issue of solving the minimum of cost functions.

It is assumed that $x \in C^{m}$ represents any complex random vector, correlation function represents $C=R_{X X}=$ $E\left\{X(n) X^{H}(n)\right\}$, cost function is $J(\mathrm{~W})$, yielding:

$$
\begin{aligned}
& J(\mathrm{~W})=E\left\{\left\|X-W W^{H} X\right\|^{2}\right\}=\operatorname{tr}(C)- \\
& 2 \operatorname{tr}\left(W^{H} C W\right)+\operatorname{tr}\left(W^{H} C W W^{H} W\right)
\end{aligned}
$$


The equitation can be obtained by the matrix-weighted method as follows:

$$
\begin{aligned}
& J(\mathrm{~W}(\mathrm{t}))=\sum_{i=1}^{t} \beta^{t-i}\left\|X(i)-W(t) W(t)^{H} X(i)\right\|^{2} \\
& =\operatorname{tr}(C(t))-2 \operatorname{tr}\left(W(t)^{H} C(t) W(t)\right) \\
& +\operatorname{tr}\left(W(t)^{H} C(t) W(t) W(t)^{H} W(t)\right)
\end{aligned}
$$

Where $W \in C^{m \times r}$ is weight matrix, if the rank can be r. It has been proved that $\mathrm{W}$ equals to eigenvector of the signal when $J(\mathrm{~W})$ reaches a minimum [6]. Therefore, the minimum of $J(\mathrm{~W})$ can be obtained by RLS algorithm, which can convert subspace decomposition into subspace iteration.

There have existed two problems in the standard PASTd algorithm: (1) The algorithm convergence velocity is sensitive to initial values. (2) The orthogonality of eigenvector is not so good.

In order to solve the above problems, we can improve the PASTd algorithm as follows:

Iterative initial value required by PASTd algorithm can be obtained by the EVD for input signals at the beginning of PASTd iteration [7].

In order to make sure better property of convergence for the algorithm, eigenvectors that are mutually orthogonal can be obtained by substituting the process of Gram-Schmidt orthogonality into PASTd algorithm.

If the rank of signal is $r$ and $W_{i}$ represents the $r$-th array of matrix $\mathrm{W}$, we may arrive at the conclusion that iterative time $\mathrm{n}$ is 0 , the maximum of iterative time is $\mathrm{D}(\mathrm{D}=\mathrm{N}-\mathrm{M}+1)$. Then, we can make the eigenvalue decomposition of autocorrelation matrix $\mathrm{C}$, that is $C=U \Lambda U^{T}=U_{s} \Lambda_{s} U_{s}{ }^{T}+U_{n} \Lambda_{n} U_{n}{ }^{T}$. At last, eigenvalue $\lambda_{i}(0)$ and eigenvector $w_{i}(0)$ can be considered as the initial values of the algorithm.

Updating the PASTd algorithm $\left\{w_{i}(n), \lambda_{i}(n)\right\}_{i=1}^{r}$

For $n=1,2,3, \cdots, D$

Do $x_{1}(n)=X(n)$

For $i=1, \cdots, r(n-1)$ Do

$y_{\mathrm{i}}(\mathrm{n})=\mathrm{w}_{\mathrm{i}}^{\mathrm{H}}(\mathrm{n}-1) x_{\mathrm{i}}(\mathrm{n})$

$\lambda_{\mathrm{i}}(\mathrm{n})=\beta \lambda_{\mathrm{i}}(\mathrm{n}-1)+\left|y_{i}(\mathrm{n})\right|^{2}$

$\mathrm{e}_{\mathrm{i}}(\mathrm{n})=x_{\mathrm{i}}(\mathrm{n})-\mathrm{w}_{\mathrm{i}}(\mathrm{n}-1) \mathrm{y}_{\mathrm{i}}(\mathrm{n})$

$\mathrm{w}_{\mathrm{i}}(\mathrm{n})=\mathrm{w}_{\mathrm{i}}(\mathrm{n}-1)+\mathrm{e}_{\mathrm{i}}(\mathrm{n})\left(\mathrm{y}_{\mathrm{i}}(\mathrm{n})\right)^{*} / \lambda_{\mathrm{i}}(\mathrm{n})$

The Gram-Schmidt arthogonality of the equation: $\mathrm{w}=\left[w_{1}(n), \cdots, w_{r}(n)\right]$

If $i \geq 2$

$$
\begin{aligned}
& \mathrm{w}=\left[w_{1}(n), \cdots, w_{i-1}(n)\right] \\
& \mathrm{w}_{\mathrm{i}}(\mathrm{n})=\mathrm{w}_{\mathrm{i}}(\mathrm{n})-w w^{H} \mathrm{w}_{\mathrm{i}}(\mathrm{n})
\end{aligned}
$$

END

$\mathrm{w}_{\mathrm{i}}(\mathrm{n})=\mathrm{w}_{\mathrm{i}}(\mathrm{n}) /\left\|\mathrm{w}_{\mathrm{i}}(\mathrm{n})\right\|$

END

$$
\lambda_{N}(n)=\beta \lambda_{N}(n-1)+\left|x_{r(t-1)+1}(n)\right|^{2} /(m-r(t-1))
$$

END

The MDL rule can be used in the eigen value order, that is $\left\{\lambda_{1}(\mathrm{n}), \ldots, \lambda_{r}(\mathrm{n}), \lambda_{r+1}(\mathrm{n})=\lambda_{N}(\mathrm{n}), \ldots, \quad \lambda_{m}(\mathrm{n})=\lambda_{N}(\mathrm{n})\right\}$ meanwhile, the rank of signal subspace $r_{s}(n)$ can be obtained by MDL rules, and update the equation $r(n)=r_{s}(n)+1$.

$\lambda_{\text {est }}=\left[\lambda_{1}+\Delta \lambda, \lambda_{2}+\Delta \lambda \ldots, \lambda_{m}+\Delta \lambda\right]$, where $\Delta \lambda=0.01 \times \lambda_{r-1}$

For $k=1,2, \cdots r(n-1)$

DO

$\alpha(k)=\left(\prod_{i=k+1}^{m} \lambda_{i}(n)^{\frac{1}{m-k}}\right) /\left[\sum_{i=k+1}^{m} \lambda_{i}(n) /(m-k)\right]$ $M D L(k)=-L(m-k) \log (\alpha(k)) /(1-\beta)+\frac{k}{2}(2 m-k) \log L$

END

$r(n)=\arg \min _{0 \leq k \leq m-1} M D L(k)+1$

Determine whether the space changes and take measures.

IF $r(n)<r(n-1)$

$\operatorname{remove}\left\{\mathrm{w}_{\mathrm{i}}(\mathrm{n}), \lambda_{\mathrm{i}}(\mathrm{n})\right\}_{i=r(n)+1}^{r(n-1)}$

ELSE IF $r(n)>r(n-1)$

$w_{r(n)}(n)=x_{r(n-1)+1}(n) /\left\|x_{r(n-1)+1}(n)\right\|$

$\lambda_{r(n)}(n)=\lambda_{N}(n)$

END W.

Based on the above, we can get the signal subspace matrix

From the above analysis, power system harmonic estimation algorithm in the paper is as follows:

Step1: After the EVD decomposition for input signals, eigenvalue and eigenvector can be considered as the initial values of the algorithm.

Step2: Subject to the improved PASTd algorithm, signal subspace matrix $\mathrm{W}$ can be obtained by the iteration from data matrix to smaller matrix, which can reduce the calculated amount of eigenvalue decomposition.

Step3: According to the improved Gram-Schmidt orthogonal algorithm, combined with the improved MDL rule, eigenvalues and eigenvectors required by ESPRIT algorithm 
can be obtained by the orthogonally for eigenvector matrix W.

Step4: Combined with ESPRIT algorithm, the harmonic frequency can be estimated.

\section{SIMULATION EXPERIMENT}

Experiment 1: in order to verify the effectiveness of the algorithm, simulated signal adopted is as follows:

$$
\begin{aligned}
& x(t)=0.3 \cos \left(2 \pi \times 25 t+70^{\circ}\right)+0.7 \cos \left(2 \pi \times 35.85 t+80^{\circ}\right)+ \\
& 1.0 \cos \left(2 \pi \times 50 t+30^{\circ}\right)+0.5 \cos \left(2 \pi \times 88.60 t+90^{\circ}\right) \\
& +0.4 \cos \left(2 \pi \times 150 t+40^{\circ}\right)+e(t)
\end{aligned}
$$

Where, sampling frequency $f_{s}=1000 \mathrm{~Hz}, S N R=80 \mathrm{~dB}$, length of window $m=100, \beta=0.99$. In order to verify the frequency estimation algorithm results, sampling order can be taken by ESPRIT estimation. The signal consists of three interharmonic frequency components $(25 \mathrm{~Hz}, 35.85 \mathrm{~Hz}, 88.6 \mathrm{~Hz})$ and two harmonic frequency components $(50 \mathrm{~Hz}, 150 \mathrm{~Hz})$. As is shown in Fig.1, the ability to estimate the interharmonics by the algorithm can be verified.

In the experiment, ESPERIT algorithm, FFT algorithm, music algorithm and this algorithm can respectively be estimated for sampling orders. The estimation results are as follows:(More specifically, Fig.1 shows waveform of the simulated signal, Fig.2, Fig3 and Fig.4 respectively show ESPERT, FFT and MUSIC analysis result.)

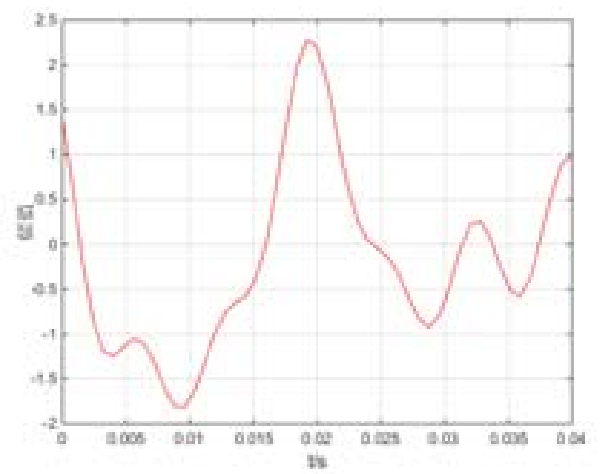

FIGURE I. WAVEFORM OF THE SIMULATED SIGNAL

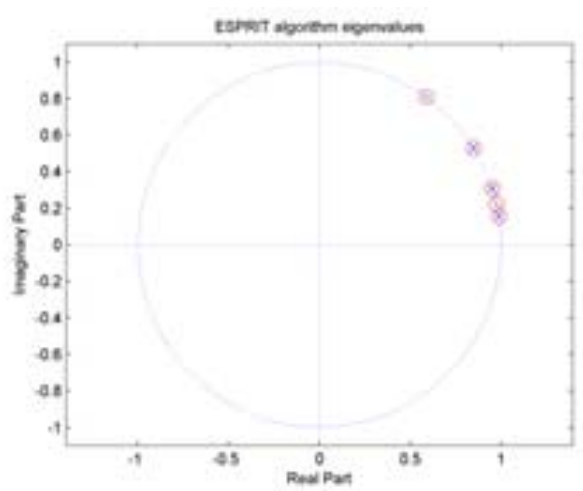

FIGURE II. ESPRIT ANALYSIS RESULT

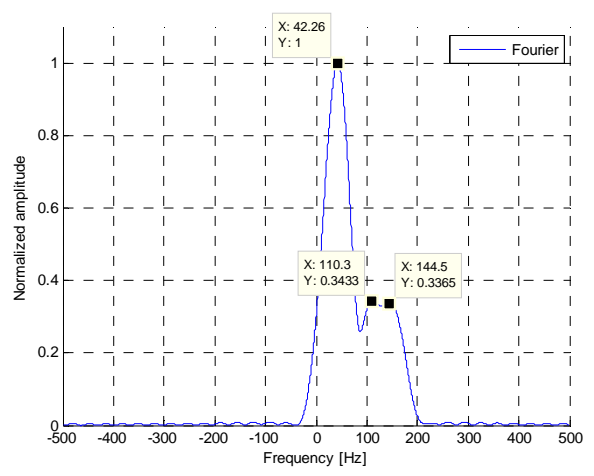

FIGURE III. FFT ANALYSIS RESULT

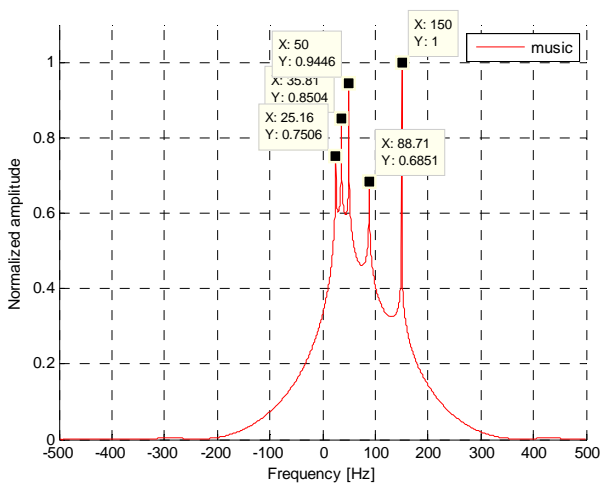

FIGURE IV. MUSIC ANALYSIS RESULT

It will be seen from the results that the harmonic frequency can be obtained by ESPRIT algorithm and music algorithm. However, compared with the algorithm adopted in the paper, the error caused by the ESPRIT and music algorithm is bigger. The peak can be shown in the spectrogram of FFT algorithm, but, the number of peak and its frequency can't be in conformity with the number of harmonic and its frequency that included in $\mathrm{x}(\mathrm{t})$. See Table 1 for the detail.

TABLE I. HARMONIC FREQUENCY ESTIMATION RESULTS IN EXPERIMENT 1

\begin{tabular}{|c|c|c|c|c|c|}
\hline $\begin{array}{c}\text { True } \\
\text { value(Hz) }\end{array}$ & 25 & 35.85 & 50 & 88.6 & 150 \\
\hline $\begin{array}{c}\text { ESPRIT } \\
\text { estimation }\end{array}$ & 24.85 & 35.96 & 50.41 & 88.85 & $\begin{array}{c}149.7 \\
3\end{array}$ \\
\hline $\begin{array}{c}\text { Relative } \\
\text { error(\%) }\end{array}$ & 0.6 & 0.31 & 0.82 & 0.282 & 0.18 \\
\hline $\begin{array}{c}\text { FFT } \\
\text { estimation }\end{array}$ & $/$ & $/$ & 42.26 & 110.3 & 144.5 \\
\hline $\begin{array}{c}\text { Relative error } \\
\text { (\%) }\end{array}$ & 100 & 100 & 15.48 & 24.49 & 3.67 \\
\hline $\begin{array}{c}\text { MUSIC } \\
\text { estimation }\end{array}$ & 25.16 & 35.81 & 50 & 88.71 & 150 \\
\hline $\begin{array}{c}\text { Relative } \\
\text { error(\%) }\end{array}$ & 0.64 & 0.11 & 0 & 0.12 & 0 \\
\hline
\end{tabular}

As indicated in Table 2, the results can be obtained by means of the improved PASTd algorithm, when different signal orders $\mathrm{r}$ are given in advance. From what we have discussed 
above, we can draw the conclusion that the algorithm discussed in the paper, which the detection precision for harmonic and interharmonic is better than that of ESPRIT and FFT algorithm, can estimate each frequency of harmonic signals exactly.

TABLE II. PASTD ALGORITHM HARMONIC FREQUENCY ESTIMATION RESULTS IN EXPERIMENT 2

\begin{tabular}{|c|c|c|c|c|c|c|}
\hline $\begin{array}{l}\text { Signal } \\
\text { order } r\end{array}$ & $\begin{array}{c}25(H \\
\mathrm{Z})\end{array}$ & $\begin{array}{c}\text { 35.85( } \\
\mathrm{Hz})\end{array}$ & $50(\mathrm{~Hz})$ & $\begin{array}{c}88.6(\mathrm{~Hz} \\
)\end{array}$ & $150(\mathrm{~Hz})$ & $\begin{array}{c}\text { Iterat } \\
\text { ion } \\
\text { time }\end{array}$ \\
\hline $\mathrm{r}=1$ & $\begin{array}{c}25.0 \\
541\end{array}$ & 38.8571 & $\begin{array}{c}50.047 \\
6\end{array}$ & 88.5842 & $\begin{array}{c}149.984 \\
5\end{array}$ & 23 \\
\hline $\mathrm{r}=2$ & $\begin{array}{c}25.0 \\
103\end{array}$ & 35.8428 & $\begin{array}{c}49.991 \\
5\end{array}$ & 88.6503 & $\begin{array}{c}150.107 \\
8\end{array}$ & 30 \\
\hline$r=3$ & $\begin{array}{l}25.1 \\
044\end{array}$ & 35.8539 & $\begin{array}{c}50.104 \\
5\end{array}$ & 88.6913 & $\begin{array}{c}150.153 \\
6\end{array}$ & 32 \\
\hline$r=4$ & $\begin{array}{c}25.2 \\
108\end{array}$ & 35.8485 & $\begin{array}{c}49.983 \\
2\end{array}$ & 88.6857 & $\begin{array}{c}150.004 \\
5\end{array}$ & 33 \\
\hline$r=5$ & $\begin{array}{c}24.9 \\
816\end{array}$ & 35.8588 & $\begin{array}{c}50.121 \\
8\end{array}$ & 88.6108 & $\begin{array}{c}149.909 \\
4\end{array}$ & 37 \\
\hline$r=6$ & $\begin{array}{l}25.0 \\
231\end{array}$ & 35.8497 & $\begin{array}{c}50.351 \\
2\end{array}$ & 88.6425 & $\begin{array}{c}150.034 \\
6\end{array}$ & 39 \\
\hline
\end{tabular}

\section{CONCLUSION}

Adopted by power system harmonic estimation, ESPRIT algorithm tends to be one of the most effective methods in frequency estimation, time delay estimation, and DOA estimation, which is characterized by higher estimation accuracy. However, due to complex calculation caused by EVD for autocorrelation matrix as well as spectral peak searching in the frequency domain, ESPRIT algorithm is not beneficial to real-time detection for harmonic PASTd algorithm can push the calculated complexity into smaller by means of iteration that converts data matrix into smaller matrix. Firstly, the signal subspace can be obtained by the orthogonality of eigenvectors that be available by the improved PASTd algorithm. At last, combined with ESPRIT algorithm, harmonic frequency can be obtained.

Combined the improved PASTd algorithm with ESPRIT algorithm, less complex calculation and better convergent performance can be obtained without affecting estimation accuracy. It has been proved that the algorithm is noted for ultrahigh resolution of harmonic estimation and better antinoise performance from the simulation results. Furthermore, the algorithm is suitable for power system harmonic estimation, which has no synchronous sampling and spectrum leakage.

\section{ACKNOWLEDGEMENTS}

This study was sponsored by National Nature Science Foundation of China (grant no.U1304501).

\section{REFERENCES}

[1] Jin Quanyi, PengAnjin. Non-integer Harmonics Parameter Measurement Algorithm Based on FFT. Computer networks ,2006; (22): 212-213

[2] Jang Yi. Based on the non-parametric method of harmonic frequency estimation research University of electronic science and technology, 2008
[3] Zhou Lin, XiaXue, WanYunjie. Harmonic Detection Based on Wavelet Transform. Transactions of China Electrotechnical Society,2006; 21(9): $67-74$

[4] Schmidt R O. Multiple emitter location and signal parameter estimates. IEEE Trans on Antennas Propagation, 1986 ; 34(3):276280

[5] Cai Guoquan,Song Guowen, Yu Dapeng. The projection approximation subspace tracking algorithm and its application in blind linear multiuser detection, Signal processing, 2000;16 (2):163-169

[6] Wang Yongliang, Chen HuiPeng, Yingning, Spatial spectral estimation theory and algorithm. Beijing: Tsinghua university press, 2004

[7] Sui Dan, Ge Lindong. A novel blind SNR estimator based on the modified PASTd algorithm for IF signals, Journal of electronics and information technology, 2007, (07): 1657-1661 